\title{
Schmerztherapie Fixkombination erspart Arzneimittelaustausch
}

Die optimale Einstellung von Patienten mit starken chronischen Schmerzen auf eine individuelle Therapie kann langwierig und aufwendig sein. Eine Option: die Fixkombination aus retardiertem Oxycodon und retardiertem Naloxon (Targin ${ }^{\circledR}$ ).

Dass eine langfristige Schmerztherapie mit dieser Fixkombination sowohl medizinisch als auch ökonomisch sinnvoll ist, belegen die Daten einer Versorgungsforschungsstudie mit 1.095 Schmerzpatienten. Sie wurden entweder mit der Fixkombination oder mit anderen starken Opioiden behandelt. Laut Reinhard Rychlik, Burscheid, ergab eine Zwischenauswertung nach sechs Monaten, dass die direkten
Kosten in der Oxycodon/Naloxon-Gruppe mit durchschnittlich 1.027 Euro pro Patient deutlich unter denen der Vergleichsgruppe (1.327 Euro) lagen. Zudem war die Steigerung der Lebensqualität stärker ausgeprägt.

In diesem Zusammenhang gab Michael Überall, Nürnberg, zu bedenken, dass ein medizinisch nicht indizierter Austausch stark wirksamer Opioid-Anal- getika gegen kostengünstigere Präparate zu einer Zunahme der Schmerzintensität und zu einer Verschlechterung der Lebensqualität führen könne. Die behandelnden Ärzte sollten bei allen Schmerzpatienten die Wirksamkeit der Therapie mithilfe von Schmerzfragebögen oder Patiententagebüchern genau dokumentieren lassen, ergänzte Gerhard Müller-Schwefe, Göppingen.

aam

Quelle: Pressegespräch am 11. März 2010 in Frankfurt/Main; Veranstalter: Mundipharma

\section{Essenzielle Thrombozythämie EWG: interdisziplinäres Management gefragt}

Die essenzielle Thrombozythämie (ET) zählt zu den myeloproliferativen Neoplasien und ist eine eher seltene chronische Erkrankung, die im Gegensatz zu vielen anderen onkologischen Indikationen häufig nicht dramatisch verläuft und den Patienten bei guter Therapie eine fast normale Überlebenszeit ermöglicht. Gerade deswegen wird ihr eher wenig Aufmerksamkeit geschenkt. Dabei stellt die Erkrankung bei Diagnose und Therapie erhebliche Anforderungen an den Hämatologen.

Neben den Symptomen und Komplikationen der ET wie Thrombosen, Blutungen und mikrovaskulärne Symptomen [Mesa RA et al. Cancer 2007;109: 68-76], können behandlungsbe-

Kurz notiert

Cetuximab bei SCCHN: EXTREME - keine Beeinträchtigung der Lebensqualität

Die Auswertung der Lebensqualitätsdaten der EXTREME-Studie ergab, dass die Verlängerung von Gesamtüberleben und progressionsfreiem Überleben durch Addition von Cetuximab zu der platinbasierten Chemotherapie bei Patienten mit rezidivierten oder metastasierten Kopf-Hals-Tumoren nicht auf Kosten der Lebensqualität erkauft wurde. Insgesamt beantworteten $80 \%$ der ITT-Population den QLQ-C30-Fragebogen [Mesia R et al. Ann Oncol. 2010; Mar 24. Epub ahead of print]. dingt offene, vielfach aber auch versteckte Nebenwirkungen wie Hautveränderungen, kardiologische oder psychiatrische Begleiterscheinungen auftreten. Obgleich sie die Lebensqualität des Patienten beeinflussen, werden sie nicht unbedingt vom Patienten oder vom Hämatologen erkannt und mit der Medikation in Zusammenhang gebracht. Diesem Problem hat sich eine von der Shire Pharmaceuticals Group initiierte interdisziplinäre Arbeitsgruppe von zehn internationalen Experten aus Hämatologie, Kardiologie, Dermatologie und Psychiatrie (Expert Working Group, EWG) gewidmet. In mehreren Workshops wurden relevante Informationen erarbeitet und mit insgesamt 40 Ärzten aus den jeweiligen Fachdisziplinen aus ganz Europa abgestimmt.

Die Ergebnisse ermöglichen dem Hämatologen ein bestmögliches ET-Management und geben Anregungen für die Vermeidung bzw. Behandlung auftretender unerwünschter Ereignisse. Ziel ist es, die kurz- und langfristigen Behandlungserfolge und die Lebensqualität der betroffenen Patienten zu verbessern.

Die erarbeiteten Inhalte werden dieses Jahr einen besonderen Schwerpunkt im Rahmen der von Shire initiierten hämatologischen Symposien einnehmen. Für weitere Informationen zur Expert Working Group oder den von Shire initiierten hämatologischen Symposien kontaktieren Sie bitte Shire Deutschland GmbH: (0 30) 2065 82-0.

Shire Deutschland 